\title{
Low expression of pro-apoptotic proteins Bax, Bak and Smac indicates prolonged progression- free survival in chemotherapy-treated metastatic melanoma
}

Cristiano Guttà', Arman Rahman ${ }^{2,3}$, Claudia Aura 2,3,4, Peter Dynoodt ${ }^{2,5}$, Emilie M. Charles, 4,6,7, Elodie Hirschenhahn ${ }^{4,6,7}$, Jesuchristopher Joseph ${ }^{2,6}$, Jasper Wouters $\mathbb{1}^{2,5,8,9}$, Ciaran de Chaumont ${ }^{2,6}$, Mairin Rafferty ${ }^{2}$, Madhuri Warren ${ }^{4}$, Joost J. van den Oord ${ }^{5}$, William M. Gallagher ${ }^{2,3}$ and Markus Rehm (1,6,7,10,11

\begin{abstract}
Despite the introduction of novel targeted therapies, chemotherapy still remains the primary treatment for metastatic melanoma in poorly funded healthcare environments or in case of disease relapse, with no reliable molecular markers for progression-free survival (PFS) available. As chemotherapy primarily eliminates cancer cells by apoptosis, we here evaluated if the expression of key apoptosis regulators (Bax, Bak, Bcl-2, Bcl-xL, Smac, Procaspase-9, Apaf-1, Procaspase-3 and XIAP) allows prognosticating PFS in stage III/IV melanoma patients. Following antibody validation, marker expression was determined by automated and manual scoring of immunohistochemically stained tissue microarrays (TMAs) constructed from treatment-naive metastatic melanoma biopsies. Interestingly and counter-intuitively, low expression of the pro-apoptotic proteins Bax, Bak and Smac indicated better prognosis (log-rank $p<0.0001, p=0.0301$ and $p=0.0227$ for automated and $p=0.0422, p=0.0410$ and $p=0.0073$ for manual scoring). These findings were independently validated in the cancer genome atlas (TCGA) metastatic melanoma cohort (TCGA-SKCM) at transcript level (log-rank $p=0.0004, p=0.0104$ and $p=0.0377$ ). Taking expression heterogeneity between the markers in individual tumour samples into account allowed defining combinatorial Bax, Bak, Smac signatures that were associated with significantly increased PFS ( $p=0.0002$ and $p=0.0028$ at protein and transcript level, respectively). Furthermore, combined low expression of Bax, Bak and Smac allowed predicting prolonged PFS (> 12 months) on a case-by-case basis (area under the receiver operating characteristic curve $(\mathrm{ROC} A \cup C)=0.79)$. Taken together, our results therefore suggest that Bax, Bak and Smac jointly define a signature with potential clinical utility in chemotherapy-treated metastatic melanoma.
\end{abstract}

\section{Introduction}

Melanoma, an aggressive neoplasm originating from the malignant transformation of melanocytes, rapidly metastasises

Correspondence: Markus Rehm (markus.morrison@izi.uni-stuttgart.de) ${ }^{1}$ Institute of Cell Biology and Immunology, University of Stuttgart, Stuttgart, Germany

${ }^{2}$ Oncomark Ltd., Nova UCD, Dublin 4, Ireland

Full list of author information is available at the end of the article. Edited by T. Kaufmann if not surgically removed at an early stage. Although novel and costly targeted treatment options and immunotherapies have significantly improved the management of metastatic disease ${ }^{1-3}$, patients in poorly funded healthcare environments still rely on chemotherapy as the primary first-line treatment. Likewise, chemotherapy remains in frequent use as a second- or last-line treatment option in otherwise refractory or in recurrent disease. Even though treatments based on the DNA-alkylating

\section{(c) The Author(s) 2020}

(c) (i) Open Access This article is licensed under a Creative Commons Attribution 4.0 International License, which permits use, sharing, adaptation, distribution and reproduction cc) in any medium or format, as long as you give appropriate credit to the original author(s) and the source, provide a link to the Creative Commons license, and indicate if changes were made. The images or other third party material in this article are included in the article's Creative Commons license, unless indicated otherwise in a credit line to the material. If material is not included in the article's Creative Commons license and your intended use is not permitted by statutory regulation or exceeds the permitted use, you will need to obtain permission directly from the copyright holder. To view a copy of this license, visit http://creativecommons.org/licenses/by/4.0/. 
agent dacarbazine have been the chemotherapeutic standard of care for metastatic melanoma for $>30$ years, chemotherapy may benefit only few patients ${ }^{4,5}$. The median survival of patients treated with dacarbazinebased chemotherapy lies in the range of 6-9 months ${ }^{6-8}$, with no reliable molecular markers available that would allow to identify those patients in which disease progression is substantially delayed and which therefore might have benefited from this treatment.

Apoptosis is the main cell death mechanism by which the body tries to eliminate transformed and therefore potentially cancerous cells. Apoptosis likewise is the primary cell death modality induced by dacarbazine and other DNA-alkylating agents. DNA alkylation induces the intrinsic apoptosis pathway, as was shown experimentally in various melanoma model systems ${ }^{9,10}$. Pro- and antiapoptotic Bcl-2 family members, such as Bax, Bak and Bcl-2, Bcl-xL, respectively, regulate the mitochondrial apoptosis signalling hub ${ }^{11}$. Activated Bax and Bak form pores in the outer mitochondrial membrane, leading to the release of pro-apoptotic factors, such as Smac, into the cytosol $^{12}$. Subsequently, the execution phase of apoptosis is initiated, during which proteases such as initiator caspase- 9 and effector caspase- 3 are activated in an Apaf1-dependent manner. These proteases then rapidly execute apoptotic death, but can be inhibited by the antiapoptotic protein XIAP, which itself is targeted by $\mathrm{Smac}^{13}$. Impaired apoptosis signalling is a hallmark of cancer ${ }^{14}$, based on which it is reasonable to assume that melanoma cells are highly apoptosis resistant. Indeed, experimental studies suggest that melanoma cells either are highly chemoresistant or acquire resistance and thereby evade apoptotic cell death ${ }^{15,16}$. However, it is less clear if perturbed expression of apoptosis regulators is indeed associated with patient prognosis in the clinical scenario. Various studies immunohistochemically assessed individual apoptosis regulators as potential protein biomarkers for melanoma progression and patient survival ${ }^{17,18}$. Unfortunately though, the majority of studies lack controls and validation information that would support the specificity of the used reagents and staining protocols. Not surprisingly, results obtained so far remained largely inconclusive or even contradictory ${ }^{17}$. Additionally, apoptosis regulators at key signalling hubs frequently act cooperatively and redundantly, so that it can be speculated that single molecule makers might not be sufficiently robust for clinical use.

In this study, we therefore assessed the expression of nine apoptosis regulators (Bax, Bak, Bcl-2, Bcl-xL, Smac, Procaspase-9, Apaf-1, Procaspase-3 and XIAP) in metastatic melanoma tissues by immunohistochemistry (IHC), using antibodies that passed rigorous validation. Interestingly, low expression of Bax, Bak and Smac associated with prolonged progression-free survival (PFS), a finding confirmed at transcriptional level in an independent cohort. Combining Bax, Bak and Smac expression with a pattern recognition approach allowed predicting individual patient PFS with high accuracy. Taken together, our results identified a putative combinatorial prognostic signature with potential clinical utility for chemotherapytreated metastatic melanoma.

\section{Materials and methods}

\section{Ethics approval and consent to participate}

The use of the patient cohort was approved by the Medical Ethical Committee and Institutional Review Board (OG032) of the University Hospitals of KU Leuven (reference number ML10659) and by the UZ Leuven Biobank (reference number S56609).

\section{Antibodies}

The following antibodies were used for immunoblotting and immunohistochemistry. A rabbit polyclonal beta Actin antibody (Santa Cruz Biotechnology; sc-81178); Apaf-1 (Cell Signalling; D5C3), Bak (Abcam; ab32371), Bax (Millipore; ABC11), Bcl-2 (Dako; MO887), Bcl-xL (BD labs; 610212), Procaspase-3 (Cell Signalling; 9662), Procaspase-9 (Cell Signalling; 9502), Smac (Cell Signalling; 2954), XIAP (BD labs; 610762).

\section{Cell culturing}

For antibody validation, the following human cancer cell lines were used: A375, HCT-116, HCT-116 (Bax/Bak) ${ }^{-1-}$, HCT-116 $\mathrm{Smac}^{-1-}$, HCT-116 XIAP ${ }^{\mathrm{o}-}$, HeLa, Jurkat Casp- $9^{-1-}$, MCF-7, PM-WK, Preyer, SK-Mel-94. Cell lines were obtained from ATCC, DSMZ or provided by colleagues (Professor Martin Leverkus, University of Heidelberg; Professor Richard Youle, National Institutes of Health, USA; Professor Richard Vogelstein, The Johns Hopkins University School of Medicine, USA; Professor Ingo Schmitz, University of Braunschweig, Germany; Professor Sebastian Wesselborg, University of Düsseldorf, Germany; Professor Maria Soengas, National Cancer Research Centre, Spain) and described before ${ }^{19-24}$. Cell lines were cultured in RPMI-1640 medium (SigmaAldrich) or Dulbecco's Modified Eagle Medium (DMEM; Lonza, Slough, UK) supplemented with $4 \mathrm{~mm}$ L-glutamine, $4.5 \mathrm{~g} / \mathrm{l}$ glucose, $10 \%(\mathrm{w} / \mathrm{v})$ heat-inactivated fetal bovine serum (Sigma-Aldrich), $100 \mathrm{U} / \mathrm{ml}$ penicillin and $100 \mu \mathrm{g} / \mathrm{ml}$ streptomycin (Sigma-Aldrich). Cells were grown at 5\% $\mathrm{CO}_{2}$ and $37^{\circ} \mathrm{C}$.

\section{Immunoblotting}

For whole cell extracts, cells were collected at $400 \mathrm{~g}$ for $3 \mathrm{~min}$ and washed with phosphate-buffered saline. Cells were re-suspended in lysis buffer $(62.5 \mathrm{~mm}$ Tris- $\mathrm{HCl}, \mathrm{pH}$ $6.8,10 \%(\mathrm{v} / \mathrm{v})$ glycerine, $2 \%(\mathrm{w} / \mathrm{v})$ sodium dodecyl sulfate (SDS), $1 \mathrm{~mm}$ phenylmethylsulfonyl fluoride, $1 \mu \mathrm{g} / \mathrm{ml}$ 
Table 1 Summary of demographics and clinical information of the patients included in the study.

\begin{tabular}{|c|c|c|}
\hline \multicolumn{3}{|l|}{ Characteristics } \\
\hline Gender & Value & $\%$ \\
\hline Male & 30 & 51.7 \\
\hline Female & 28 & 48.3 \\
\hline Age at surgery (years) & Value & $\%$ \\
\hline$<65$ & 44 & 75.9 \\
\hline$\geq 65$ and $<75$ & 8 & 13.8 \\
\hline$>75$ & 6 & 10.3 \\
\hline Metastatic melanoma location & Value & $\%$ \\
\hline Distant skin site & 10 & 17.2 \\
\hline Distant organ & 17 & 29.3 \\
\hline Distant lymph node & 28 & 48.3 \\
\hline Distant subcutaneous site & 3 & 5.2 \\
\hline Metastasis stage & Value & $\%$ \\
\hline M1a & 8 & 13.8 \\
\hline $\mathrm{M} 1 \mathrm{~b}$ & 8 & 13.8 \\
\hline M1c & 42 & 72.4 \\
\hline Primary melanoma type & Value & $\%$ \\
\hline Cutaneous & 46 & 79.3 \\
\hline Mucosal & 1 & 1.7 \\
\hline Ocular & 2 & 3.4 \\
\hline Unknown & 9 & 15.5 \\
\hline Treatment & Value & $\%$ \\
\hline Dacarbazine & 3 & 5.2 \\
\hline Dacarbazine, Cisplatin & 54 & 93.1 \\
\hline Dacarbazine, Carboplatin & 1 & 1.7 \\
\hline Overall survival & $\begin{array}{l}t_{0}=\text { sample } \\
\text { collection }\end{array}$ & $\begin{array}{l}t_{0}=\text { chemotherapy } \\
\text { start }\end{array}$ \\
\hline Median (range) in months & $19(2-126)$ & $11(0-87)$ \\
\hline Progression-free survival & $\begin{array}{l}t_{0}=\text { sample } \\
\text { collection }\end{array}$ & $\begin{array}{l}t_{0}=\text { chemotherapy } \\
\text { start }\end{array}$ \\
\hline Median (range) in months & $10(1-100)$ & $4(0-83)$ \\
\hline
\end{tabular}

pepstatin A, $1 \mu \mathrm{g} / \mathrm{ml}$ leupeptin, and $5 \mu \mathrm{g} / \mathrm{ml}$ aprotinin) and heated at $95^{\circ} \mathrm{C}$ for $20 \mathrm{~min}$. Protein content was determined with the Pierce Micro-BCA protein assay (Pierce, Northumberland, UK). An equal amount of protein $(20 \mu \mathrm{g})$ was loaded onto SDS-polyacrylamide gels. Proteins were separated at $100 \mathrm{~V}$ for $2.5 \mathrm{~h}$ and then blotted to nitrocellulose membranes (Protean BA 83; $2 \mu \mathrm{m}$; Schleicher \& Schuell) in transfer buffer ( $25 \mathrm{~mm}$ Tris,
$192 \mathrm{~mm}$ glycine, $20 \%$ methanol (v/v), and $0.01 \%$ SDS) at $18 \mathrm{~V}$ for $60 \mathrm{~min}$. The blots were blocked with $5 \%$ non-fat dry milk in Tris-buffered saline with Tween 20 (TBST) $(15 \mathrm{~mm}$ Tris- $\mathrm{HCl}, \mathrm{pH} 7.5,200 \mathrm{~mm} \mathrm{NaCl}$, and $0.1 \%$ Tween 20) at room temperature for $1 \mathrm{~h}$. Membranes were incubated with the primary antibodies at room temperature for $2 \mathrm{~h}$ or overnight at $4^{\circ} \mathrm{C}$. Membranes were washed with TBST three times for $5 \mathrm{~min}$ and incubated with peroxidase-conjugated secondary antibodies (Jackson Laboratories) for $1 \mathrm{~h}$. Blots were washed and developed using the enhanced chemiluminescence detection reagent (Millipore, Ireland).

\section{Preparation of cell pellets for IHC}

Cells were grown to a confluence of $50-75 \%$. Cells were then detached and suspended in 10\% phosphate-buffered formalin at room temperature and fixed for 4-6h. Fixed cells were centrifuged at $500 \times g$ for $3 \mathrm{~min}$, washed once with $1 \times \mathrm{PBS}$ and pelleted again. A $1 \%$ agarose solution was prepared in $1 \times \mathrm{PBS}$ and cooled down to $40^{\circ} \mathrm{C}$ in a water bath. The cell/agarose mixtures were transferred into plugs and let solidify. The agarose plugs were processed into paraffin blocks using standard tissue processing. Cell pellet samples (typically $0.6 \mathrm{~mm}$ in diameter) were then used for analysis.

\section{Tissue microarrays (TMAs)}

TMAs of formalin-fixed paraffin-embedded (FFPE) tumour samples derived from 74 melanoma patients treated with Dacarbazine (alone or in combination with cisplatin or carboplatin), were generated. The TMA contained duplicate cores obtained from 14 primary melanomas, 62 metastatic melanomas and adjacent normal tissue. Demographics, clinical and follow-up information were available for the entire cohort. A total of $n=$ 58 samples, representing untreated metastatic melanoma patients, were analysed for this study (Table 1).

\section{Immunohistochemistry}

IHC staining on FFPE cell pellets and tissue microarrays (TMA) was performed using an automated IHC platform (Link-48, Dako, Glostrup, Denmark) according to the manufacturer's instructions. Sections $(4 \mu \mathrm{m}$ in thickness) were deparaffinised and antigen retrieval was performed at $95^{\circ} \mathrm{C}$ for $15 \mathrm{~min}$ in appropriate buffer (high pH buffer, pH 9.0; low pH buffer, pH 6.0) using the PT-Link module (Dako, Glostrup, Denmark). A polymer-based detection system (EnVision Flex, Dako) was used with Permanent Red as the chromogen, resulting in a red colour endpoint that contrasted well with brown melanin. Sections were counterstained with haematoxylin. Positive and negative controls (omission of the primary antibody and replacement with the IgG-2a isotype control, mouse-ab18443; IgG isotype control, rabbit-ab208334, Abcam, Cambridge, 
UK) were included in each run. In addition, a Haematoxylin and Eosin (H\&E) staining was performed for all slides of the TMAs, enabling pathologists to check for TMA core integrity, quality and tumour content.

\section{Core quality assessment}

A pathological review of the H\&E-stained sections and TMA blocks was conducted to define the quality of individual tissue cores and to assess the percentage of tumour tissue in each core. Each core was individually observed to determine whether there were any tissue artifacts (poorly fixed tissue, folded tissue, no tumour present, no tissue present, foreign material introduced at embedding, poor tissue microscopic details) or staining artifacts (knife marks across section, holes, clumps of stain precipitate, air bubbles), which would have compromised either the manual or automated image analysis. All quality assessments were independently validated by a second pathologist. Cores with compromising artifacts or with insufficient percentage of tumour cells were excluded from further analyses.

\section{Manual and automated scoring}

IHC materials were first viewed at low power to judge overall quality and distribution of staining. Subsequently, staining frequency (total \% stained cells) and staining intensity (intensity of stained cells; $0=$ no staining, $1+=$ weak staining, $2+=$ moderate staining, $3+=$ strong staining) were determined. Histoscores ( $H$ scores) were then calculated as follows:

$$
\text { Hscore }=1 \times \%_{\text {cells } 1+}+2 \times \%_{\text {cells } 2+}+3 \times \%_{\text {cells } 3+}
$$

The manual scoring was performed on images acquired with the Aperio ScanScope XT slide scanner (Aperio Technologies, Vista, CA) used at $\times 20$ magnification with a maximum pixel resolution of $0.5 \mu \mathrm{m}$. ImageScope analysis software (Aperio Technologies, Vista, CA) was used for viewing and analysing digital images. Aperio Spectrum software was used to generate individual tissue spot images for automated analysis. The Colour Deconvolution algorithm (Aperio Technologies) was used to obtain quantitative values for average positive intensity (average intensity of pixels positively stained, graded from $0,1,2,3$ ) and total percent positive (percentage of positive stained area in relation to total area of the core). Histoscores were calculated as described above.

\section{Survival analysis}

PFS was calculated as the time between the surgery that procured the sample and the date of disease progression or of a new metastatic event in a different location. Pathologist's and automated $H$ score were used to separate patients with high (above median) and low (below median) expression of each marker protein included in this study. In case more than one tissue core with satisfactory quality was available for a single patient, the average $H$ score was considered. Log-rank testing was used to compare the two groups over a follow-up time of 36 months. Log-rank testing for trends was used when comparing three groups. Kaplan-Meier survival curves were generated and compared using GraphPad Prism (version 4.03). For analysis of data stored in the cancer genome atlas (TCGA), normalised mRNA expression data (upper quartile normalised Fragments per Kilobase of transcript per million mapped reads, $\log _{2}$ (FPKM-UQ + 1)) generated by the Genomic Data Commons (GDC$\mathrm{NIH}$ ) were downloaded from the UCSC-XENA brow$\operatorname{ser}^{25,26}$. The SKCM cohort, unlike other TCGA data sets, contains mainly metastatic samples ${ }^{27}$ (370 out of 477), some of which were collected a long time after initial diagnosis of the primary melanoma ${ }^{28}$. In order to correlate mRNA expression to progression of metastatic disease, the 'new tumour event free survival' was calculated as the time between sample collection and the first new tumour event (in case of multiple new tumour events during the follow-up time) or, in case of no new tumour events, death. If a new tumour event was reported before the date of sample collection, the patient was excluded from the sub-cohort. Follow-up data and associated clinical records were downloaded from Broad GDAC Firehose $^{29}$ (new tumour event time from initial diagnosis) and UCSC-XENA browser ${ }^{25,26}$ (overall survival from initial diagnosis), respectively. Sample collection information are available through the GDC data portal ${ }^{30,31}$ (time from initial diagnosis to sample collection). As treatment information are not routinely available for all deposited metastatic melanoma cases, we downselected the cohort to stage III/IV melanoma patients diagnosed with metastatic melanoma before 2010, to ensure that chemotherapy-based treatment options would have been the standard first line of treatment ( $n=79$ patients). An optimised chi-square-based cutoff was determined to divide patients with high and low $B A X, B A K 1$ and DIA$B L O$ (Smac) mRNA amounts, and the two groups were compared by log-rank test. The cutoff for each marker was obtained by selecting the cohort separation that resulted in the highest chi-squared value with the function survdiff of the library survival in R (version 3.4.0). Median cutoff-based results are reported in Supplementary Fig. 4. Log-rank test for trend was used when comparing three groups. Kaplan-Meier survival curves were generated and compared using GraphPad Prism (version 4.03).

\section{Data-driven modelling and pattern recognition}

A data-driven modelling approach based on a previously published method ${ }^{32}$ was developed to predict patients' PFS using $H$ scores generated by automated image 
analysis as input. The pipeline was developed for MATLAB (version 2016a, The Mathworks, UK), equipped with the statistical toolbox. Prior to the analysis, patients with a complete protein panel $(n=50)$ were divided into two PFS categories: PFS $>12$ months $(n=17)$ and PFS $<$ 12 months $(n=33)$. After standardisation of the initial data set, a principal component analysis (PCA) was performed and the principal components (PC) with an eigenvalue $>1$ were considered for subsequent analyses. The patients were positioned in the 3D space defined by the first three PCs according to the scores computed by PCA, and linear discriminant analysis (LDA) was used to test the class segmentation accuracy. To evaluate the predictive potential of the framework, leave one out cross validation (LOOCV) followed by LDA was applied iteratively to the data set, using 49 patients as training set and one patient as test at each iteration. LDA was also applied to a data set reduced to three proteins (Bax, Bak and Smac), skipping the initial dimensionality reduction step. PCA and LDA were performed using the functions $p c a$ and classify, respectively. The predictive performance of the two classification models was compared by computing the area under the curve (AUC) with the function perfcurve.

\section{Results}

Low expression of pro-apoptotic proteins Bax, Bak and Smac correlates with increased PFS in chemotherapytreated metastatic melanoma

Genotoxic chemotherapy based on DNA-alkylating agents such as dacarbazine induces intrinsic apoptosis, preferentially in proliferating cells such as cancer cells. Intrinsic apoptosis is governed by the family of Bcl-2 proteins and the subsequent signalling network of the apoptosis execution phase. We therefore tested key players of this apoptosis signalling modules as potential prognostic markers in metastatic melanoma. In total, we analysed the expression of six pro-apoptotic (Bax, Bak, Smac, Procaspase-9, Apaf-1, Procaspase-3) and three antiapoptotic proteins (Bcl-2, Bcl-xL, XIAP) in metastatic melanoma samples spotted on TMAs. Only treatmentnaive samples from metastases were used for subsequent analyses. Information such as patient demographics, histopathology and staging, treatment and follow-up are provided as Supplementary Table 1 and are summarised in Table 1. Following comprehensive antibody validation (Supplementary Fig. 1A-D), IHC stains for $n=58$ tumour metastases matching the inclusion criteria were analysed from the TMAs. Only tissue samples passing independent pathologist quality control for tissue integrity and staining artifacts were considered for subsequent analyses (Supplementary Table 2). TMAs scans were then used to generate mark-up images of the tissue cores, followed by automated quantification of staining intensities (see methods). The dynamic range of the staining intensities allowed to confidently define quartiles of negative, low, medium and high staining for protein expression (see Fig. 1a for examples for Bax, Bak and Smac). From these, $H$ scores were calculated for each tumour sample (Fig. 1b), thereby allowing comparison with best practice manual scoring (see Fig. 2). To test if protein expression amounts and patient prognosis correlate, we performed survival analyses for all nine apoptosis regulatory proteins. Kaplan-Meier curves representing PFS from the date of sample procurement showed that low amounts of proapoptotic proteins Bax, Bak and Smac significantly correlated with better prognosis (Fig. 1c). With the exception of Procaspase-9, which associated with better prognosis in this analysis, none of the other proteins $(\mathrm{Bcl}-2, \mathrm{Bcl}-\mathrm{xL}$, Apaf-1, XIAP and Procaspase-3) individually correlated with better or worse prognosis (Supplementary Fig. 2). Overall, these results surprisingly indicate that low amounts of apoptosis-inducing proteins Bax, Bak and Smac are linked to a better prognosis in chemotherapytreated metastatic melanoma.

\section{Manual scoring confirms association of low Bak, Bax and Smac protein expression with improved PFS}

To further validate our findings, we next conducted best practice manual scoring of the stained TMAs. $H$ scores for all marker candidates were obtained from two independent pathologists, both blinded to patient PFS. Plotting $H$ scores obtained by automated analysis against manual $H$ scores, we noted that manual scores strongly clustered at values of $\sim 200$, whereas automated scoring provided higher granularity across the entire dynamic range (Fig. 2a, Fig. 1b, Supplementary Table 2). This highlights that manual scoring appears limited in differentiating within the range of medium staining intensities and frequencies. Nevertheless, median separation of patient samples based on manual $H$ scores provided survival curves for Bax, Bak and Smac staining that were very similar to those obtained by automated scoring (Fig. 2b). In contrast, the manual scores for all other proteins failed to separate patients with high and low PFS (Supplementary Fig. 3). These results therefore demonstrate that the Bax, Bak and Smac signatures are robust enough to also be captured in routine manual IHC-based biomarker discovery workflows.

\section{Combined low expression of Bax, Bak and Smac is a combinatorial marker candidate for improved PFS}

During apoptosis, Bax and Bak form pores in the outer mitochondrial membrane, leading to Smac release into the cytosol. Owing to the significant correlation of the single proteins with PFS and their direct relationship within the apoptosis signal transduction cascade, we checked if combinations of the three markers could 


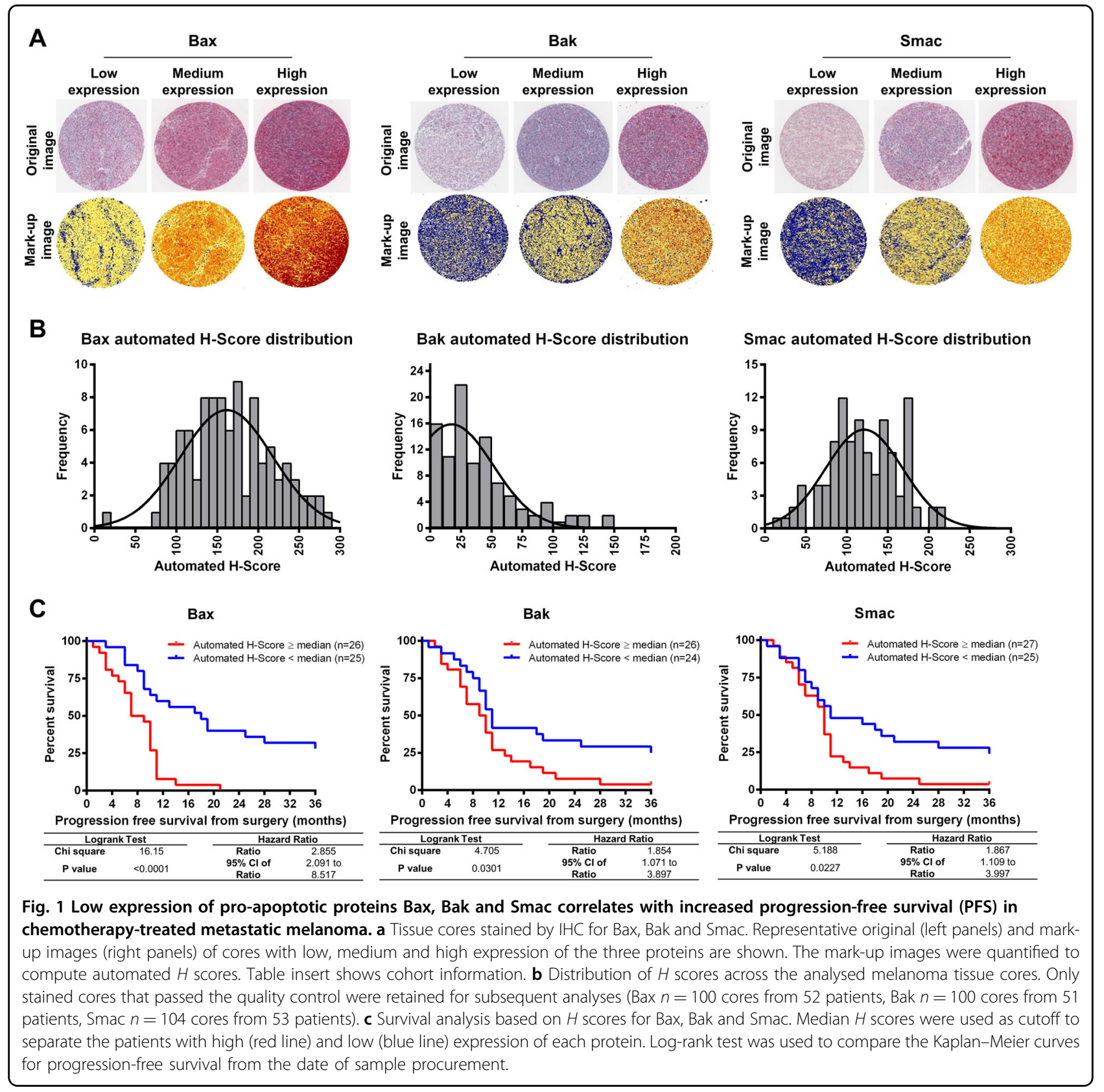

improve prognostication of PFS. For the $n=50$ patients for which $H$ scores for Bax, Bak and Smac were available, we noted that combined low or high staining for all three markers was restricted to subsets of the tumour samples (Fig. 3a). We therefore divided the cohort into three groups of combined high expression, heterogeneous expression and combined low expression. PFS-based survival analysis of the three groups demonstrated that patients harbouring tumours with combined low expression of Bax, Bak and Smac showed significantly improved PFS, extending beyond 36 months for $50 \%$ of this subgroup (Fig. 3b). In contrast, when only one or two markers where expressed in low amounts, PFS improved only slightly (median PFS $=10$ months vs. 8.5 months when all three markers were highly expressed) (Fig. 3b). Overall, this shows that Bax, Bak and Smac could jointly define a signature that strongly associates with PFS, with combined low expression indicating improved PFS.

\section{TCGA-SKCM-based analysis validates the prognostic Bax, Bak, Smac signature}

To independently validate the prognostic potential of Bax, Bak and Smac expression, we analysed transcriptome data of $n=79$ metastatic melanoma patients from the TCGA-SKCM cohort (Table 2). The survival analysis revealed that low $B A X, B A K 1$ and DIABLO 

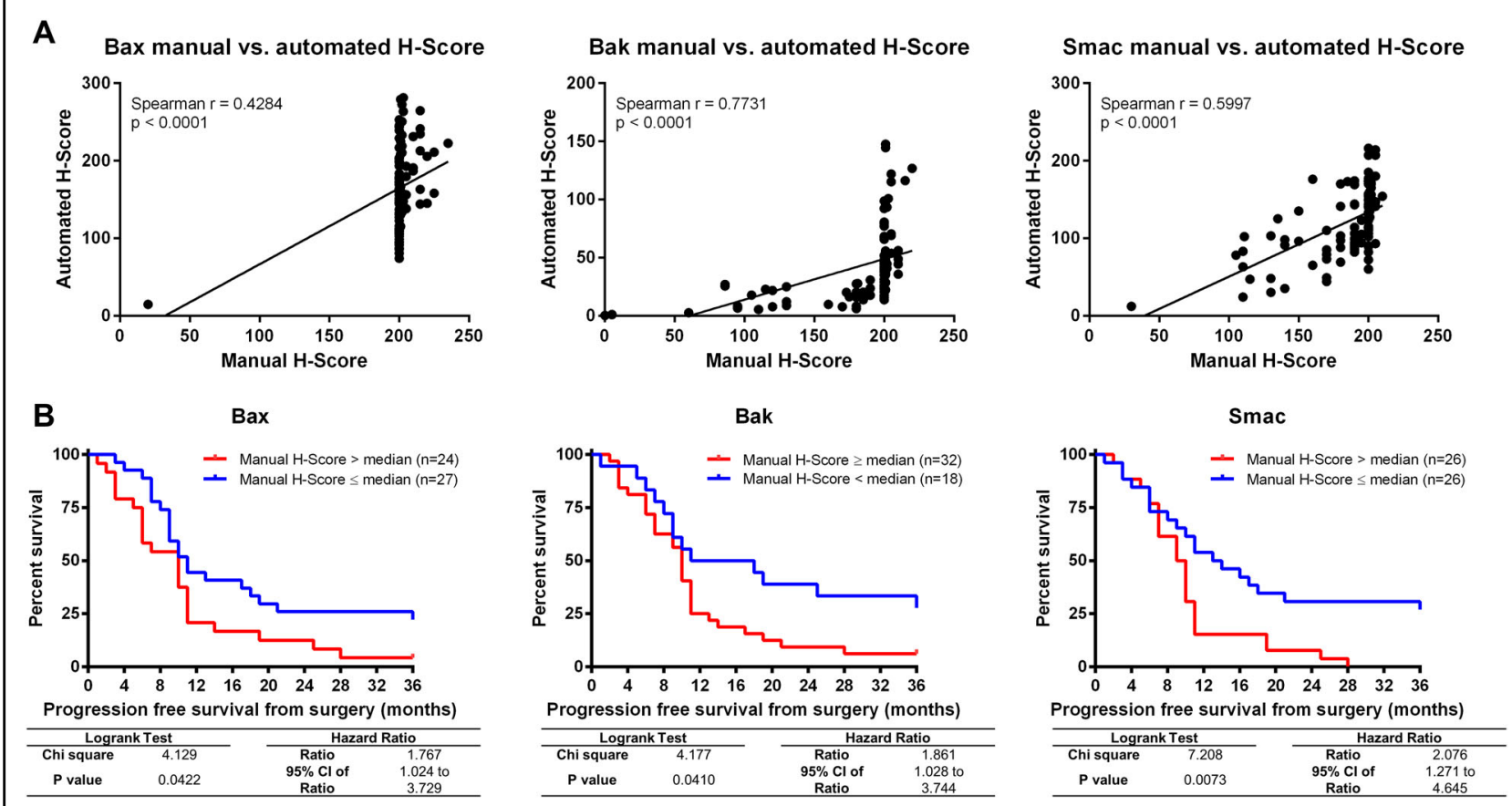

Fig. 2 Manual scoring confirms association of low Bak, Bax and Smac protein expression with improved PFS. a Correlation between automated and manual $\mathrm{H}$ scores. Manual scores were obtained from two independent pathologists blinded to patient follow-up. Correlation was analysed using Spearman's rank correlation coefficient. b Median $\mathrm{H}$ scores were used as cutoff to separate the patients with high (red line) and low (blue line) expression of each protein. Log-rank test was used to compare the Kaplan-Meier curves for progression-free survival from the date of sample procurement.

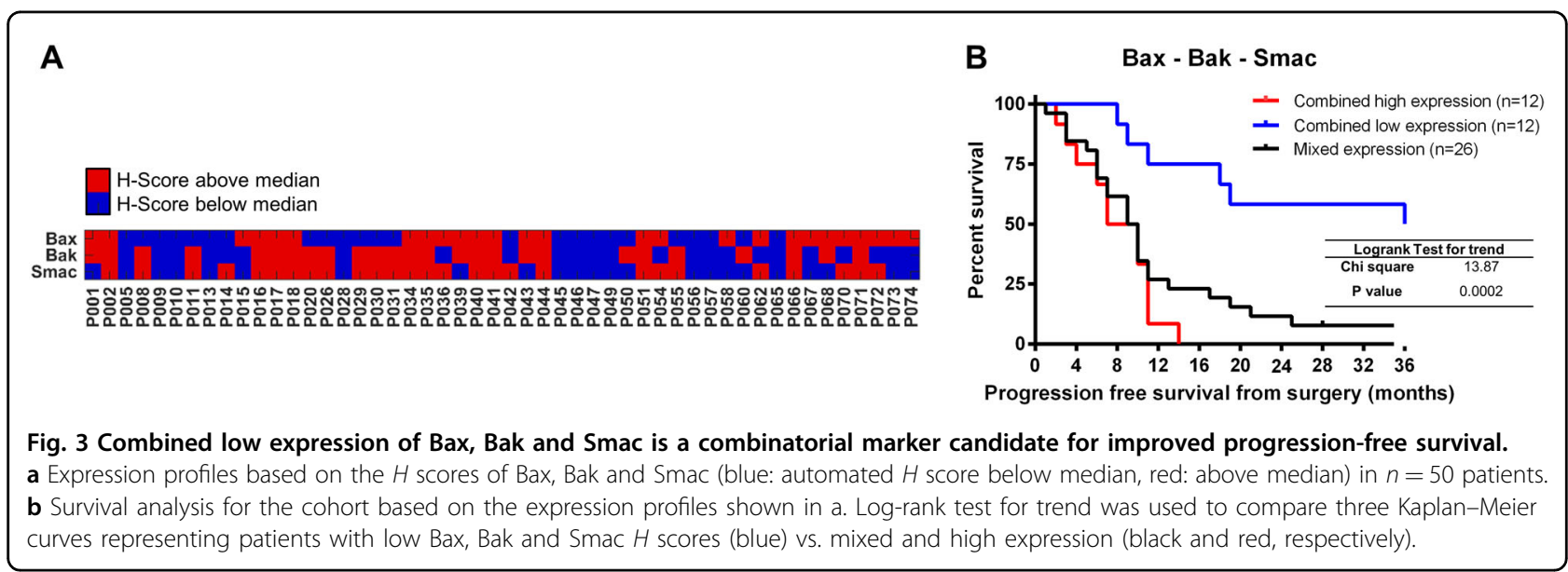

$(S M A C)$ mRNA amounts significantly correlate with better prognosis (Fig. 4a). As previously observed at protein level, the expression pattern between $B A X$, $B A K 1$ and DIABLO was heterogeneous across the cohort (Fig. 4b). Patients with low tumour mRNA amounts across all three markers had a significantly better prognosis than patients in which at least one marker was highly expressed (Fig. 4c). Taken together, these results recapitulate in an independent cohort the trends observed at protein level, confirming the prognostic potential of Bax, Bak and Smac as a combinatorial marker in chemotherapy-treated metastatic melanoma.

\section{Pattern recognition allows predicting patient prognosis}

We next applied a data-driven pattern recognition approach to study if the Bax, Bak, Smac signature would be sufficiently strong to predict patient PFS from protein expression profiles ${ }^{32}$. First, $H$ scores from automated TMA analysis for all marker candidates were subjected to 
Table 2 Patient demographics and clinical information of the metastatic SKCM-TCGA sub-cohort.

\begin{tabular}{lll}
\hline Characteristics & & \\
\hline Gender & Value & $\%$ \\
Female & 32 & 40.51 \\
Male & 47 & 59.49 \\
Ethnicity & Value & $\%$ \\
White (non-Hispanic or Latino) & 79 & 100 \\
Disease stage at initial diagnosis & Value & \\
Stage III & 68 & \\
Stage IV & 11 & \\
Age at diagnosis (years) & & \\
Mean & 55.5 & \\
Median & 55 & \\
Range & $18-87$ \\
Overall survival from initial diagnosis (months) & \\
Mean & 35.1 & \\
Range & $2.6-175.2$ & \\
Overall survival from sample procurement (months) & \\
Mean & 15.9 & \\
Range & $1.1-64.9$ & \\
\hline
\end{tabular}

a PCA (applied to the 50 patient samples for which the complete nine protein panel was available). Patient tumours were positioned into a 3D space defined by the first three PCs and colour-coded to represent high or low PFS (PFS $>12$ months and PFS $<12$ months). Visually inspecting the scatter plot, we noticed a tendency of patients with high or low PFS to occupy distinct subregions of the PC space (Fig. 5a). To objectively assess the quality of this segregation, we applied LDA. LDA segmentation encouragingly separated $72 \%$ of the patients into their correct prognosis sub-space. Next, we tested if these patterns were sufficiently strong to also predict the PFS category on a case-by-case basis. To do so, we performed leave one out cross validation (LOOCV). At each iteration, the PFS category of one patient was predicted after using the remaining 49 patients as a training set that defined the PCA subspaces for high and low PFS. The panel of nine apoptosis regulatory proteins allowed to correctly predict high or low PFS in $74 \%$ of patients. Since our previous survival analyses (Fig. 1) showed that only Bax, Bak and Smac consistently correlated with PFS, we likewise tested if a similarly good or even better performing classifier can be derived from those three markers alone. Indeed, cluster segmentation and prediction accuracy tended to improve to $80 \%$ and $78 \%$ accuracy, respectively (Fig. 5b). In conclusion, as highlighted by the comparison of receiver operating characteristic (ROC) curves (Fig. 5c), classification based on the Bax-Bak-Smac signature alone is sufficient to obtain high prediction accuracies for patient PFS, whereas the remaining protein markers do not carry meaningful information to improve these predictions. Overall, this strengthens the evidence for low Bax, Bak and Smac expression being associated with better prognosis in metastatic melanoma and points out a route by which pattern recognition allows generating predictions for patient prognosis.

\section{Discussion}

Apoptosis is the major cell death modality by which anticancer therapies eliminate malignant neoplastic cells. In this study, we assessed if proteins that regulate the two major apoptosis decision hubs, namely the apoptotic engagement of mitochondria and the terminal execution phase of apoptosis ${ }^{33}$, alone or in combination can serve to prognosticate PFS in metastatic melanoma patients undergoing dacarbazine-based chemotherapy. We found that low rather than high expression of the pro-apoptotic proteins Bax, Bak and Smac correlates with higher PFS, and that these three proteins in combination can serve as a combinatorial prognostic marker with a promising AUC of 0.79 .

Owing to the central role of apoptosis in tumour cell elimination, the finding that low expression of proapoptotic proteins correlated with better prognosis in metastatic melanoma contradicted our expectations. However, counter-intuitive relationships between the expression patterns of apoptosis inducers or antiapoptotic genes or proteins were reported previously. For example, high expression of Bax was found to correlate with an increased risk for relapse in childhood acute lymphoblastic leukaemia ${ }^{34}$. High expression of Bax, measured as transcript and protein amounts, respectively, has also been associated with poor prognosis in acute myeloid leukaemia and non-Hodgkin lymphoma ${ }^{35,36}$. Similarly, studies in which high expression of the Bax antagonist Bcl-2 has been reported to correlate with better prognosis can be found for colorectal, breast, glioma, gastric and non small cell lung cancer ${ }^{37-43}$. Bax and Bcl-2 are the best-characterised members of the Bcl-2 protein family, which controls mitochondrial engagement in apoptosis signal transduction ${ }^{44,45}$, whereas Bak has been less thoroughly studied. Bak functions as a Bax-like protein and upon activation likewise is able to form pores in the outer mitochondrial membrane, thereby triggering apoptosis execution $^{44,45}$. Links between low Bak expression and an improved outcome have not been reported in metastatic melanoma so far, but reduced $B A K$ mRNA amounts were associated with better overall survival in hepatocellular carcinoma ${ }^{46}$. Similarly, a counter-intuitive prognostic value of Smac has not yet been reported in melanoma, but 

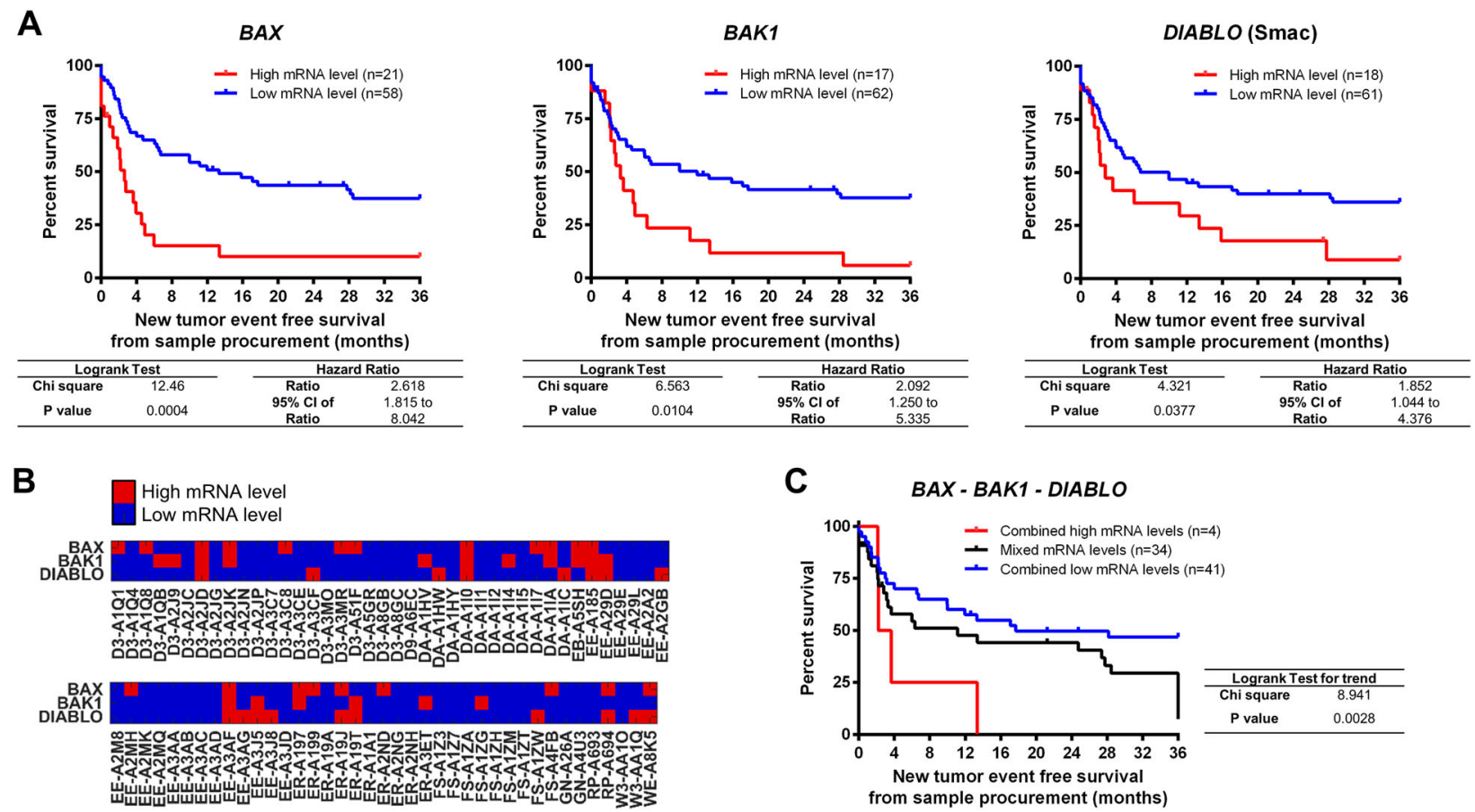

Fig. 4 TCGA-SKCM-based analysis validates the prognostic Bax, Bak, Smac signature. Independent validation of the prognostic signature at transcriptome level in the SKCM-TCGA metastatic sub-cohort. a Survival analysis in the SKCM-TCGA sub-cohort $(n=79$ patients diagnosed with stage III or IV metastatic melanoma before 2010). An optimised chi-square-based cutoff was determined to divide patients with high (red) and low (blue) normalised BAX, BAK1 and DIABLO (Smac) expression ( $\log _{2}(F P K M-U Q+1)$ ). Kaplan-Meier curves (follow-up from sample procurement) were compared by log-rank test. b mRNA amounts for BAX, BAK1 and DIABLO (Smac) (blue: mRNA level below cutoff, red: mRNA level above cutoff). c Survival analysis in the metastatic TCGA-SKCM sub-cohort based on the expression profiles in Fig. 4b. Log-rank test for trend was used to compare three Kaplan-Meier curves representing patients with combined low BAX, BAK1 and DIABLO (Smac) expression (blue), combined high expression (red) or with mixed expression (black).

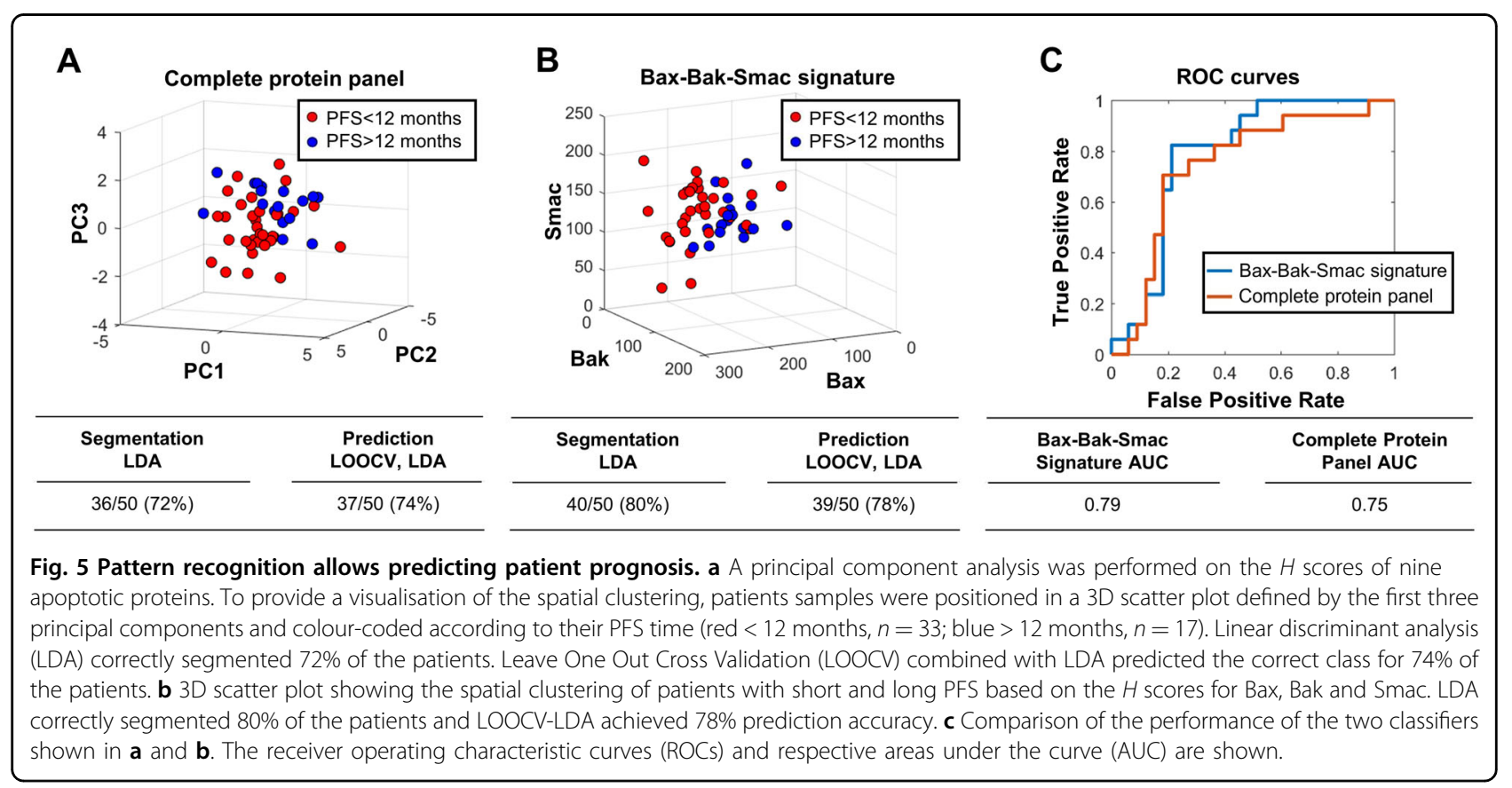


high expression was found to correlate with early local disease recurrence in cervical cancer ${ }^{47}$. It needs to, however, be stated that in reverse a large body of literature associates high expression of pro-apoptotic or a low expression of antiapoptotic genes or proteins with better outcome, as would intuitively be expected (see e.g., ref. ${ }^{48-59}$ ). Overall, it therefore appears that signatures indicative of apoptosis competency or resistance need to be interpreted or studied within the specific disease setting and context. For example, it was suggested that expression patterns indicative of high apoptosis responsiveness may correlate with poor outcome if dormant, stem-like cancer cells that may reside within tumour tissues re-populate tumours and promote further spread and progression of the disease after the bulk population of cells has been eliminated by apoptosisinducing therapy ${ }^{42,43}$. In line with this, apoptotic cell loss can drive the proliferation of surrounding cells, for example, through caspase-dependent prostaglandin signalling and secretion of other proliferation stimulating factors from dying cells ${ }^{60,61}$. These signalling processes indeed might be of relevance in melanoma treatment responsiveness and disease relapse ${ }^{62}$.

As apoptosis resistance is a hallmark of cancer ${ }^{63}$, it nevertheless appears puzzling that reduced expression of apoptosis drivers correlates with better prognosis in a treatment scenario that is clearly geared towards apoptosis induction. In addition to the above line of thoughts, the very high mutation burden of cutaneous melanoma ${ }^{64}$ might provide the basis for an additional explanation. Although unfavourable expression of key apoptosis regulators in many cases may cause apoptotic cell death to be suppressed during cell transformation, tumour development and disease progression, and as such could be considered causative for the disease, such low basal apoptosis susceptibilities might nevertheless be overcome by elevated apoptosis-inducing stress in chemotherapy settings. In contrast, where low apoptosis susceptibility is not causative for the disease (and hence protein expression profiles would indicate "normal" susceptibility), other alterations and mutations might drive the development and progression of the disease. Many of these could prevent therapy-induced stress signals to be channelled towards apoptosis induction. Indeed, low expression of Bax and Bak might be linked to disease progression in earlier stages of melanoma. Although Bax protein expression tends to be higher in melanoma tissues than in benign nevi ${ }^{65}$, low expression of Bax within primary superficial-spreading melanoma was associated with poor prognosis and therefore could indicate a role in disease development and progression ${ }^{66}$. Similar findings were reported for Bak expression in the same study. Also in stage IIa melamoma, low Bax and Bak protein expression was associated with poor prognosis, with the majority of such patients developing metastatic disease ${ }^{67}$. Taken together, these prior reports combined with our findings therefore suggest that low expression of pro-apoptotic players could be causative for early stage tumour formation and melanoma disease progression by lowering basal apoptosis susceptibility, and that this reduced susceptibility can be overcome once pro-apoptotic stress is elevated externally, for example by dacarbazine-based chemotherapy. It will be interesting to see if similar relationships can also be found in other cancer (sub)types in the future.

\begin{abstract}
Acknowledgements
We kindly acknowledge support by the European Union's Horizon 2020 research and innovation programme under the Marie Skłodowska-Curie grant agreement \#642295 (MEL-PLEX) and by the European Union's FP7 Marie Skłodowska-Curie Industry-Academia Partnership and Pathways research programme under the grant agreement \#611107 (SYS-MEL). M.R. and C.G. are funded by the Deutsche Forschungsgemeinschaft (DFG, German Research Foundation) under Germany's Excellence Strategy - EXC 2075 - 390740016. M.R. also receives support from the German Research Foundation (FOR2036 (MO 3226/1-1)) and the Health Research Board Ireland (HRA POR 2015 1091). J. $W$. received a postdoctoral research fellowship from Kom op tegen Kanker (Stand up to Cancer), the Flemish Cancer Society, and is currently funded by a postdoctoral fellowship from Stichting tegen Kanker (Foundation against Cancer), the Belgian Cancer Society.
\end{abstract}

\section{Author details}

${ }^{1}$ Institute of Cell Biology and Immunology, University of Stuttgart, Stuttgart, Germany. ${ }^{2}$ Oncomark Ltd., Nova UCD, Dublin 4, Ireland. ${ }^{3}$ UCD School of Biomolecular and Biomedical Science, UCD Conway Institute, University College Dublin, Dublin 4, Ireland. ${ }^{4}$ Pathology Diagnostics Ltd., Stirling House Business Centre, Waterbeach, Cambridge, UK. ${ }^{5}$ Translational Cell and Tissue Research, KU Leuven (University of Leuven), Leuven, Belgium. ${ }^{6}$ Department of Physiology and Medical Physics, Royal College of Surgeons in Ireland, Dublin 2, Ireland. ${ }^{7}$ Centre for Systems Medicine, Royal College of Surgeons in Ireland, Dublin 2, Ireland. ${ }^{8}$ VIB-KU Leuven Center for Brain \& Disease Research, KU Leuven, Leuven, Belgium. ${ }^{9}$ Department of Human Genetics, KU Leuven (University of Leuven), Leuven, Belgium. ${ }^{10}$ Stuttgart Research Center Systems Biology, University of Stuttgart, Stuttgart, Germany. ${ }^{11}$ Stuttgart Center for Simulation Science (SC SimTech), University of Stuttgart, Stuttgart, Germany

Competing interests

The authors declare that they have no conflict of interests.

\section{Publisher's note}

Springer Nature remains neutral with regard to jurisdictional claims in published maps and institutional affiliations.

Supplementary Information accompanies this paper at (https://doi.org/ 10.1038/s41419-020-2309-3).

Received: 5 December 2019 Revised: 29 January 2020 Accepted: 30 January 2020

Published online: 13 February 2020

\section{References}

1. Heppt, M. V. et al. The systemic management of advanced melanoma in 2016. Oncol. Res. Treat. 39, 635-642 (2016).

2. Margolin, K. The promise of molecularly targeted and immunotherapy for advanced melanoma. Curr. Treat. Options Oncol. 17, 1-14 (2016).

3. Domingues, B., Lopes, J., Soares, P. \& Populo, H. Melanoma treatment in review. ImmunoTargets Ther. 7, 35-49 (2018).

4. Lui, P. et al. Treatments for metastatic melanoma: synthesis of evidence from randomized trials. Cancer Treat. Rev. 33, 665-680 (2007). 
5. Gupta, A., Gomes, F. \& Lorigan, P. The role for chemotherapy in the modern management of melanoma. Melanoma Manag 4, 125-136 (2017).

6. Middleton, M. R. et al. Randomized phase III study of temozolomide versus dacarbazine in the treatment of patients with advanced metastatic malignant melanoma. J. Clin. Oncol. 18, 158-158 (2000).

7. Bedikian, A. Y. et al. Bcl-2 antisense (oblimersen sodium) plus dacarbazine in patients with advanced melanoma: the oblimersen melanoma study group. $J$. Clin. Oncol. 24, 4738-4745 (2006).

8. Atkins, M. B. et al. Phase III trial comparing concurrent biochemotherapy with cisplatin, vinblastine, dacarbazine, interleukin-2, and interferon alfa-2b with cisplatin, vinblastine, and dacarbazine alone in patients with metastatic malignant melanoma (e3695): a trial coordinated by the Eastern Cooperative Oncology Group. J. Clin. Oncol. 26, 5748-5754 (2008).

9. Anvekar, R. A. et al. Sensitization to the mitochondrial pathway of apoptosis augments melanoma tumor cell responses to conventional chemotherapeutic regimens. Cell Death Dis. 3, 1-11 (2012).

10. Sanada, M. et al. Modes of actions of two types of anti-neoplastic drugs, dacarbazine and ACNU, to induce apoptosis. Carcinogenesis 28, 2657-2663 (2007).

11. Czabotar, P. E., Lessene, G., Strasser, A. \& Adams, J. M. Control of apoptosis by the BCL-2 protein family: implications for physiology and therapy. Nat. Rev. Mol. Cell Biol. 15, 49-63 (2014).

12. Tait, S. W. G. \& Green, D. R. Mitochondria and cell death: outer membrane permeabilization and beyond. Nat. Rev. Mol. Cell Biol. 11, 621-632 (2010).

13. Hellwig, C. T., Passante, E. \& Rehm, M. The molecular machinery regulating apoptosis signal transduction and its implication in human physiology and pathophysiologies. Curr. Mol. Med. 11, 31-47 (2011).

14. Hanahan, D. \& Weinberg, R. A. Hallmarks of cancer: the next generation. Cell 144, 646-674 (2011)

15. Soengas, M. S. \& Lowe, S. W. Apoptosis and melanoma chemoresistance. Oncogene 22, 3138-3151 (2003).

16. Lev, D. C. et al. Exposure of melanoma cells to dacarbazine results in enhanced tumor growth and metastasis in vivo. J. Clin. Oncol. 22, 2092-2100 (2004).

17. Charles, E. M. \& Rehm, M. Key regulators of apoptosis execution as biomarker candidates in melanoma. Mol. Cell Oncol. 1, e964037 (2014).

18. Anvekar, R. A., Asciolla, J. J., Missert, D. J. \& Chipuk, J. E. Born to be alive: a role for the BCL-2 family in melanoma tumor cell survival. Apoptosis Treat. Front Oncol. 1, 1-16 (2011).

19. Cummins, J. M. et al. X-linked inhibitor of apoptosis protein (XIAP) is a nonredundant modulator of tumor necrosis factor-related apoptosis-inducing ligand (TRAIL)-mediated apoptosis in human cancer cells. Cancer Res. 64, 3006-3008 (2004).

20. Geserick, P. et al. Suppression of CFLIP is sufficient to sensitize human melanoma cells to TRAlL- and CD95L-mediated apoptosis. Oncogene $\mathbf{2 7}$ 3211-3220 (2008).

21. Kohli, M. et al. SMAC/Diablo-dependent apoptosis induced by nonsteroidal antiinflammatory drugs (NSAIDs) in colon cancer cells. Proc. Natl Acad. Sci. USA 101, 16897-16902 (2004).

22. Samraj, A. K., Keil, E., Ueffing, N., Schulze-Osthoff, K. \& Schmitz, I. Loss of caspase-9 provides genetic evidence for the type ///l concept of CD95mediated apoptosis. J. Biol. Chem. 281, 29652-29659 (2006).

23. Soengas, M. S. et al. Inactivation of the apoptosis effector Apaf-1 in malignant melanoma. Nature 409, 207-211 (2001).

24. Wang, C. \& Youle, R. J. Predominant requirement of Bax for apoptosis in HCT116 cells is determined by Mcl-1's inhibitory effect on Bak. Oncogene 31, 3177-3189 (2012).

25. UCSC Xena. Available at: https://xena.ucscedu/. (Accessed 24 Oct 2018).

26. Goldman, M. et al. The UCSC Xena platform for public and private cancer genomics data visualization and interpretation. bioRxiv 326470 (2019). https:// doi.org/10.1101/326470.

27. Liu, J. et al. An integrated TCGA pan-cancer clinical data resource to drive high-quality survival outcome analytics. Cell 173, 400-416.e11 (2018).

28. Akbani, R. et al. Genomic classification of cutaneous melanoma. Cell 161, 1681-1696 (2015).

29. Broad Institute TCGA Genome Data Analysis Center. Analysis-ready standardized TCGA data from Broad GDAC Firehose 2016 01 28 run. Broad Institute of MIT and Harvard (2016). https://doi.org/10.7908/C11GOKM9.

30. GDC Data Portal. Available at: https://portal.gdc.cancer.gov/. (Accessed 25 Oct 2018).

31. Grossman, R. L. et al. Toward a shared vision for cancer genomic data. N. Engl. J. Med. 375, 1109-1112 (2016).
32. Passante, E., Würstle, M. L., Hellwig, C. T., Leverkus, M. \& Rehm, M. Systems analysis of apoptosis protein expression allows the case-specific prediction of cell death responsiveness of melanoma cells. Cell Death Differ. 20, 1521-1531 (2013).

33. Taylor, R. C., Cullen, S. P. \& Martin, S. J. Apoptosis: controlled demolition at the cellular level. Nat. Rev. Mol. Cell Biol. 9, 231-241 (2008).

34. Hogarth, L. A., Hall, A. G. \& Increased, B. A. X. expression is associated with an increased risk of relapse in childhood acute lymphocytic leukemia. Blood $\mathbf{9 3}$, 2671-2678 (1999).

35. Köhler, T. et al. High Bad and Bax mRNA expression correlate with negative outcome in acute myeloid leukemia (AML). Leukemia 16, 22-29 (2002).

36. Bairey, O., Zimra, Y., Shaklai, M., Okon, E. \& Rabizadeh, E. BCl-2, BCl-X, Bax, and Bak expression in short- and long-lived patients with diffuse large B-cell lymphomas. Clin. Cancer Res. 5, 2860-2866 (1999).

37. Meterissian, $\mathrm{S}$. $\mathrm{H}$. et al. $\mathrm{BCl}-2$ is a useful prognostic marker in Dukes' $\mathrm{B}$ colon cancer. Ann. Surg. Oncol. 8, 533-537 (2001).

38. Vargas-Roig, L. M. et al. Prognostic value of $\mathrm{BCl}-2$ in breast cancer patients treated with neoadjuvant anthracycline based chemotherapy. Mol. Oncol. 2, 102-111 (2008).

39. McDonald, F. E. et al. The prognostic influence of bcl-2 in malignant glioma. Br. J. Cancer 86, 1899-1904 (2002).

40. Inada, T., Kikuyama, S., Ichikawa, A., Igarashi, S. \& Ogata, Y. Bcl-2 expression as a prognostic factor of survival of gastric carcinoma. Anticancer Res. 18, 2003-2010 (1998).

41. Renouf, D. J. et al. BCL-2 expression is prognostic for improved survival in nonsmall cell lung cancer. J. Thorac. Oncol. 4, 486-491 (2009).

42. Labi, V. \& Erlacher, M. How cell death shapes cancer. Cell Death Dis. 6, e1675 (2015).

43. Ichim, G. \& Tait, S. W. G. A fate worse than death: apoptosis as an oncogenic process. Nat. Rev. Cancer 16, 539-548 (2016).

44. Hantusch, A., Rehm, M. \& Brunner, T. Counting on death - quantitative aspects of BCl-2 family regulation. FEBS J. 285, 4124-4138 (2018).

45. Kale, J., Osterlund, E. J. \& Andrews, D. W. BCL-2 family proteins: changing partners in the dance towards death. Cell Death Differ. 25, 65-80 (2018).

46. Kong, X. et al. ZBP-89 and Sp1 contribute to Bak expression in hepatocellular carcinoma cells. BMC Cancer 18, 419 (2018).

47. Arellano-Llamas, A. et al. High Smac/DIABLO expression is associated with early local recurrence of cervical cancer. BMC Cancer 6, 256 (2006).

48. Leverkus, M. \& Gollnick, H. 'Bak (and Bax) to the future' - of primary melanoma prognosis? J. Investig. Dermatol. 126, 1212-1214 (2006).

49. Kupryjańczyk, J. et al. Evaluation of clinical significance of TP53, BCL-2, BAX and MEKI expression in 229 ovarian carcinomas treated with platinum-based regimen. Br. J. Cancer 88, 848-854 (2003).

50. Baekelandt, M., Holm, R., Nesland, J. M., Tropé, C. G. \& Kristensen, G. B. Expression of apoptosis-related proteins is an independent determinant of patient prognosis in advanced ovarian cancer. J. Clin. Oncol. 18, 3775-3781 (2000).

51. Del Poeta, G. et al. Amount of spontaneous apoptosis detected by Bax/BCl-2 ratio predicts outcome in acute myeloid leukemia (AML). Blood 101, 2125-2131 (2003).

52. Jeong, S. H. et al. Low expression of bax predicts poor prognosis in resected non-small cell lung cancer patients with non-squamous histology. Jpn. J. Clin. Oncol. 38, 661-669 (2008).

53. Seok, Y. K. et al. Low expression of bax predicts poor prognosis in patients with locally advanced esophageal cancer treated with definitive chemoradiotherapy. Clin. Cancer Res. 13, 4146-4153 (2007).

54. $\mathrm{Ma}$, J. et al. Bromodomain-containing protein 7 sensitizes breast cancer cells to paclitaxel by activating Bcl2-antagonist/killer protein. Oncol. Rep. 41, 1487-1496 (2019)

55. Luo, Y. et al. High Bak expression is associated with a favorable prognosis in breast cancer and sensitizes breast cancer cells to paclitaxel. PLOS ONE 10 e0138955 (2015).

56. Endo, K. et al. Clinical significance of Smac/DIABLO expression in colorectal cancer. Oncol. Rep. 21, 351-355 (2009).

57. Dobrzycka, B. et al. Prognostic significance of smac/DIABLO in endometrioid endometrial cancer. Folia Histochem. Cytobiol. 48, 678-681 (2010).

58. Grzybowska-lzydorczyk, O., Cebula, B., Robak, T. \& Smolewski, P. Expression and prognostic significance of the inhibitor of apoptosis protein (IAP) family and its antagonists in chronic lymphocytic leukaemia. Eur. J. Cancer 46, 800-810 (2010) 
59. Pluta, P. et al. Correlation of Smac/DIABLO protein expression with the clinicopathological features of breast cancer patients. Neoplasma 58, 430-435 (2011)

60. Huang, Q. et al. Caspase 3-mediated stimulation of tumor cell repopulation during cancer radiotherapy. Nat. Med. 17, 860-866 (2011).

61. Zhao, R. et al. Novel roles of apoptotic caspases in tumor repopulation, epigenetic reprogramming, carcinogenesis, and beyond. Cancer Metastasis Rev. 37, 227-236 (2018).

62. Donato, A. L. et al. Caspase 3 promotes surviving melanoma tumor cell growth after cytotoxic therapy. J. Invest. Dermatol. 134, 1686-1692 (2014).

63. Hanahan, D. \& Weinberg, R. A. Hallmarks of cancer: the next generation. Cell 144, 646-674 (2011)
64. Lawrence, M. S. et al. Mutational heterogeneity in cancer and the search for new cancer-associated genes. Nature 499, 214-218 (2013).

65. Tang, L. et al. Expression of apoptosis regulators in cutaneous malignant melanoma. Clin. Cancer Res. 4, 1865-1871 (1998).

66. Fecker, L. F. et al. Loss of proapoptotic BCl-2-related multidomain proteins in primary melanomas is associated with poor prognosis. J. Invest. Dermatol. 126 1366-1371 (2006)

67. Tchernev, G. \& Orfanos, C. E. Downregulation of cell cycle modulators p21, p27, p53, Rb and proapoptotic Bcl-2-related proteins Bax and Bak in cutaneous melanoma is associated with worse patient prognosis: preliminary findings. J. Cutan. Pathol. 34, 247-256 (2007). 